\title{
Understanding the Changes in Diagnostic Criteria for Functional Constipation in Pediatric Patients: From Rome III to Rome IV
}

\author{
Yoo Jin Lee and Kyung Sik Park* \\ Department of Internal Medicine, Keimyung University School of Medicine, Daegu, Korea
}

Article: Functional chronic constipation: Rome III criteria versus Rome IV criteria

Russo M, Strisciuglio C, Scarpato $E$, et al

(J Neurogastroenterol Motil 2019;25:123-128)

Functional constipation (FC) is a common functional gastrointestinal disorder (FGID), with an estimated prevalence of up to $29.6 \%$ of children worldwide. ${ }^{1,2}$ Symptoms related with FC can cause distress to the quality of life of children and their families and significantly increase health care utilization and medical costs. ${ }^{3}$ Although FC is a major pediatric disease with a high prevalence, its diagnosis is still challenging. Due to lack of specific biomarkers and structural abnormalities, the diagnosis of FC relies on medical history based on the Rome criteria and physical examinations. ${ }^{4}$ Unlike the adult population, young children often have difficulties reporting their symptoms properly. This is the main difficulty in diagnosing $\mathrm{FC}$ in this age group.

In 2016, the Rome IV criteria were released, replacing the Rome III criteria, which were published in $2006 .{ }^{5,6}$ Subtle changes were adapted in the Rome IV criteria compared with the Rome III criteria, for the diagnosis of FC. ${ }^{4}$ The most notable changes incorporated into the Rome IV criteria for FC include the following: First, a differentiation has been made between toilet-trained and non-toilet-trained children. Two diagnostic criteria were eliminated for children under the age of 4 years who are not toilet trained. This was done to avoid unnecessary assessment. For example, the criteria "large-diameter stools that may obstruct a toilet" is not suitable for this age group and the assessment of "fecal incontinence with at least one episode a week" is not reliable in infants and toddlers who wear diapers. ${ }^{6,7}$ Second, the time criterion for the diagnosis has been modified from 2 months to 1 month, because a 2-month interval may delay diagnosis in some patients. ${ }^{4,6,7}$ As a consequence of the subtle revision of the existing criteria, the Rome IV criteria are expected to make the early diagnosis of FC in pediatric populations easier.

Although the Rome IV criteria have been updated based on high-quality scientific data and suggested as a gold standard for the diagnosis of FGID, their usefulness should be demonstrated in the real clinical setting. Due to some changes in the criteria, it is possible that a subset of patients who were previously defined as having FC, will no longer fit in the FC category according to the Rome IV criteria. From this point of view, evaluating the agreement between Rome III and IV criteria for FC diagnosis is crucial in coordinating future research using the Rome IV criteria and prior studies according to the Rome III criteria. ${ }^{8}$ However, there is paucity of data

\section{Received: November 30, 2018 Revised: None Accepted: December 9, 2018}

↔ This is an Open Access article distributed under the terms of the Creative Commons Attribution Non-Commercial License (http://creativecommons. org/licenses/by-nc/4.0) which permits unrestricted non-commercial use, distribution, and reproduction in any medium, provided the original work is properly cited.

${ }^{*}$ Correspondence: Kyung Sik Park, MD Division of Gastroenterology and Hepatology, Department of Internal Medicine, Keimyung University School of Medicine, 56 Dalseongro, Jung-gu, Daegu 41931, Korea

Tel: +82-53-250-7088, Fax: +82-53-250-7442, E-mail: seenae99@dsmc.or.kr 
regarding the clinical use of the Rome IV criteria to diagnose FC compared with the Rome III criteria.

In this issue of the Journal of Neurogastroenterology and Motility, Russo et al, ${ }^{9}$ published a prospective, longitudinal study in which the authors established the agreement between the Rome III and Rome IV criteria for the diagnosis of FC. Moreover, they investigated the effect of the two related questionnaires on establishing the prevalence of FC. The updated Rome IV criteria showed good agreement with the Rome III criteria for the diagnosis of FC, without an increase in the number of potential diagnoses, despite the reduction in the duration of the symptoms. Specifically, overall $18.2 \%$ of children fulfilled the Rome IV criteria for FC, while $17.3 \%$ of children fulfilled the Rome III criteria for FC. This result suggested that the updates to the Rome IV diagnostic criteria do not increase the false positive diagnosis of $\mathrm{FC}$, but are expected to accelerate earlier diagnoses. This is a meaningful result supporting the purpose of the revised Rome criteria for FC.

What is the clinical significance of the early diagnosis of FC in children? According to previous literature, delayed diagnosis of childhood FC may lead to poor clinical outcomes. ${ }^{10,11}$ A recent study also suggested that diagnosing an FGID at the first visit was the only significant prognostic factor associated with symptom resolution. ${ }^{12}$ Thus, early and adequate diagnoses are essential for appropriate treatment in patients with $\mathrm{FC}$, ultimately leading to better outcomes in these patients.

It is noteworthy that this study investigated the Rome IV questionnaires in an unselected outpatient population. Few studies have evaluated the prevalence of FC using the Rome criteria and most of them were conducted in secondary and tertiary hospitals, ${ }^{12-14}$ while the majority of children suffering from constipation are present and managed in a primary care setting. Moreover, the diagnosis of FC was made not only based on a questionnaire, but also included a physical examination in this study. The retrospective chart review or community-based survey performed without meeting physicians may have some bias and increased chances of false diagnoses. Thus, the subjects included in this study may represent general children and adolescents attending the primary medical care facility for FC.

In summary, Russo et $\mathrm{al}^{9}$ evaluated the implications of updating the Rome diagnostic criteria for FC. Changing the diagnostic criteria from Rome III to Rome IV did not increase the prevalence of $\mathrm{FC}$ in the pediatric population, despite the reduction in the symptom duration. These findings may be valuable to clinicians and researchers utilizing the Rome criteria. However, it remains undetermined whether the changes to the diagnostic criteria for FC in Rome IV ultimately impact the diagnostic stability and clini- cal outcomes in clinical practice. Further longitudinal studies are encouraged for confirming the usefulness of the Rome IV criteria in diagnosing pediatric FC, which can envisage new attempts at improving the Rome criteria.

\section{Financial support: None.}

Conflicts of interest: None.

Author contributions: Yoo Jin Lee drafted and edited the manuscript and Kyung Sik Park performed critical revision and final approval of this article.

\section{References}

1. Mugie SM, Benninga MA, Di Lorenzo C. Epidemiology of constipation in children and adults: a systematic review. Best Pract Res Clin Gastroenterol 2011;25:3-18.

2. Rajindrajith S, Devanarayana NM. Constipation in children: novel insight into epidemiology, pathophysiology and management. J Neurogastroenterol Motil 2011;17:35-47.

3. Liem O, Harman J, Benninga M, Kelleher K, Mousa H, Di Lorenzo C. Health utilization and cost impact of childhood constipation in the United States. J Pediatr 2009;154:258-262.

4. Koppen IJ, Nurko S, Saps M, Di Lorenzo C, Benninga MA. The pediatric Rome IV criteria: what's new? Expert Rev Gastroenterol Hepatol 2017;11:193-201.

5. Rasquin A, Di Lorenzo C, Forbes D, et al. Childhood functional gastrointestinal disorders: child/adolescent. Gastroenterology 2006;130:15271537.

6. Benninga MA, Faure C, Hyman PE, St James Roberts I, Schechter NL, Nurko S. Childhood functional gastrointestinal disorders: neonate/ toddler. Gastroenterology Published Online First: 15 Feb 2016. doi: 10.1053/j.gastro.2016.02.016

7. Robin SG, Keller C, Zwiener R, et al. Prevalence of pediatric functional gastrointestinal disorders utilizing the Rome IV criteria. J Pediatr 2018;195:134-139.

8. Edwards T, Friesen C, Schurman JV. Classification of pediatric functional gastrointestinal disorders related to abdominal pain using Rome III vs. Rome IV criterions. BMC Gastroenterol 2018;18:41.

9. Russo M, Strisciuglio C, Scarpato E, et al. Functional chronic constipation: Rome III criteria versus Rome IV criteria. J Neurogastroenterol Motil 2019;25:123-128.

10. van den Berg MM, van Rossum CH, de Lorijn F, Reitsma JB, Di Lorenzo C, Benninga MA. Functional constipation in infants: a follow-up study. J Pediatr 2005;147:700-704

11. Bongers ME, van Wijk MP, Reitsma JB, Benninga MA. Long-term prognosis for childhood constipation: clinical outcomes in adulthood. Pediatrics 2010;126:e156-e162.

12. Trivic I, Hojsak I. Initial diagnosis of functional gastrointestinal disorders 
in children increases a chance for resolution of symptoms. Pediatr Gastroenterol Hepatol Nutr 2018;21:264-270.

13. Osatakul S, Puetpaiboon A. Use of Rome II versus Rome III criteria for diagnosis of functional constipation in young children. Pediatr Int
2014;56:83-88.

14. Xin HW, Fang XC, Zhu LM, et al. Diagnosis of functional constipation: agreement between Rome III and Rome II criteria and evaluation for the practicality. J Dig Dis 2014;15:314-320. 